\title{
Electronic coupling mechanisms and characteristics for optically nonlinear photoactive nanomaterials
}

\author{
David L. Andrews* and David S. Bradshaw \\ Nanostructures and Photomolecular Systems, School of Chemical Sciences, \\ University of East Anglia, Norwich, NR4 7TJ, U.K.
}

\begin{abstract}
In a range of nanophotonic energy harvesting materials, resonance energy transfer (RET) is the mechanism for the intermolecular and intramolecular transfer of electronic excitation following the absorption of ultraviolet/visible radiation. In the nonlinear intensity regime, suitably designed materials can exhibit two quite different types of mechanism for channeling the excitation energy to an acceptor that is optically transparent at the input frequency. Both mechanisms are associated with two-photon optical excitation - of either a single donor, or a pair of donor chromophores, located close to the acceptor. In the former case the mechanism is two-photon resonance energy transfer, initiated by two-photon absorption at a donor, and followed by RET directly to the acceptor. The probability for fulfilling the initial conditions for this mechanism (for the donors to exhibit two-photon absorption) is enhanced at high levels of optical input. In the latter twin-donor mechanism, following initial one-photon excitations of two electronically distinct donors, energy pooling results in a collective channeling of their energy to an acceptor chromophore. This mechanism also becomes effective under high intensity conditions due to the enhanced probability of exciting donor chromophores within close proximity of each other and the acceptor. In this paper we describe the detailed balance of factors that determines the favored mechanism for these forms of optical nonlinearity, especially electronic factors. Attention is focused on dendrimeric nanostar materials with a propensity for optical nonlinearity.
\end{abstract}

Keywords: Dendrimers, energy transfer, light harvesting, multiphoton absorption, nanophotonics, nonlinear optics

\section{INTRODUCTION}

The elucidation of detailed principles for electronic energy flow in multichromophore polymers ${ }^{1}$ has led to a proliferation of new energy-harvesting materials tailored for a host of nanophotonic applications. ${ }^{2-4}$ An extensive range of multiply-branched polymers known as dendrimers, and other schematically related multichromophore assemblies, prove highly effective in the capture of optical radiation, reflecting a multiplicity of antenna chromophores and the high efficiency of RET mechanisms for intramolecular channeling of optically acquired energy to an acceptor core. Energy capture and storage applications range from uses as photosensitisers in the laser photodynamic therapy of cancer ${ }^{5,6}$ to devices based on organic light-emitting diodes. ${ }^{7,8}$ Increasingly, attention is being focused on applications associated with response to laser input and the associated high levels of irradiation. Already, the principles of two-photon fluorescence RET are involved in quite distinct areas such as two-photon three-dimensional imaging ${ }^{9-10}$ - a technique with well-known advantages for biological specimens due to reduced scatter, enhanced depth profiling and a reduction of photolytic damage resulting from the facility to employ long wavelengths. Here we focus on the energy transfer mechanisms of optically nonlinear photoactive materials, with a particular focus on dendrimeric systems.

Under conditions of high optical flux, suitably designed dendrimeric polymers can exhibit two quite different types of mechanism for channeling excitation energy to an acceptor that is optically transparent at the input frequency. ${ }^{11-14}$ Each mechanism is initiated by the absorption of two photons within the peripheral, antenna region of the dendrimer. This may result in the optical excitation of either a single donor, or a pair of donor chromophores. The first mechanism ${ }^{15-18}$ involves a twin-donor process which, following one-photon excitations of two separate and electronically distinct 
chromophores $\left(\mathrm{A}\right.$ and $\mathrm{A}^{\prime}$ ), results in energy pooling i.e. a collective migration of donor energies to the acceptor chromophore, $\mathrm{A}^{*}+\mathrm{B}+\mathrm{A}^{\prime *} \stackrel{\text { Pooling }}{\longrightarrow} \mathrm{A}+\mathrm{B}^{*}+\mathrm{A}^{\prime}$. The second mechanism ${ }^{19,20}$ entails initial two-photon absorption by a single chromophore $\mathrm{A}$, and subsequently proceeds by two-photon resonance energy transfer (TPRET) directly to a neighbouring acceptor $B$, i.e. $A^{* *}+B \stackrel{\text { TPRET }}{\longrightarrow} A+B^{*}$, where the two-star superscript denotes a two-photon excited state. In previous work it has been established that high intensity laser input is equally effective in fulfilling the conditions for each mechanism. Energy pooling and TPRET can compete, if each is allowed, since they both lead from the same initial state to a final state in which the acceptor is electronically excited. However these two mechanisms cannot display quantum interference, due to the differences in the patterns of energy dissipation and dephasing through internal vibrational redistribution (IVR) in the donor ensemble.

To expedite future progress in the design and development of optically nonlinear light-harvesting systems, it is clearly necessary to ascertain the means of differentiating, optimizing and exploiting the mechanisms for energy capture. Since the mechanisms that are available to mediate energy harvesting under conditions of high photon flux differ markedly from those available at lower intensities, a primary aim is to secure a thorough understanding of the principles that apply to systems specifically designed for operation at high levels of laser intensity, and the balance of factors and constraints that determine the favoured mechanism for each form of optical nonlinearity. This work identifies the principal factors.

\section{COOPERATIVE AND ACCRETIVE TRANSFER: ENERGY POOLING PROCESSES}

As illustrated by figure 1 the fundamental process of energy pooling accommodates; ${ }^{16}$ (a) cooperative transfer, where initial one-photon excitations in each of two neighboring donors are followed by RET from both donors directly to the acceptor, and (b) accretive transfer, where the initial excitation energy of one donor is passed to a partner donor and the sum of the two excitations transfers to the acceptor. A theoretical representation based on molecular quantum electrodynamics (QED) can be used to derive an expression for the overall efficiency of energy pooling, accommodating both transfer mechanisms. To resolve the rate equations, we need to consider the detailed nature of the RET step and statistical features associated with the initial excitation and donor conditions. Determining factors are the number of donors contained within the laser focal volume, the probability of two such donors being excited over a common period of time (through not requiring simultaneity of excitation), and the efficiency of energy transfer to the acceptor i.e. the complex RET step that completes the mechanism.
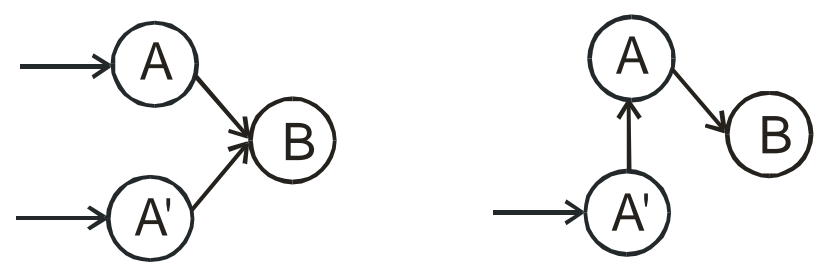

Fig. 1. Cooperative transfer (left) and accretive (right). A and $\mathrm{A}^{\prime}$ are interchangeable; arrows denote the energy transfer.

First consider the probability of satisfying the initial conditions for excitation of a donor pair. Let $N_{A}$ be the number of donors at the laser focus; the number of pairs is $1 / 2 N_{A}\left(N_{A}-1\right)$, and if $N_{1 A}$ signifies the number of donors in the relevant electronic excited state, the probability of both partners in any one pair being excited is $\left(N_{1 A} / N_{A}\right)^{2}$, assuming the decay lifetime is short compared to the laser pulse duration. Under such steady-state conditions we have;

$$
\frac{N_{1 A}}{N_{A}}=\frac{k_{A}^{1 \leftarrow 0}}{k_{A}^{1 \leftarrow 0}+k_{A}^{0 \leftarrow 1}}
$$


where $k_{A}^{0 \leftarrow 1}$ is the de-excitation rate constant and $k_{A}^{1 \leftarrow 0}$ is the absorption rate constant for the donor chromophore $A$ (dependent on the input intensity at the appropriate frequency). Application of molecular QED leads to the following result, cast in terms of molecular properties;

$$
k_{A}^{1 \leftarrow 0}=\frac{\pi I}{3 \hbar c \varepsilon_{0}}\left|\boldsymbol{\mu}^{\alpha 0(A)}\right|^{2} \rho_{f}^{A^{*}} .
$$

Here $I$ is the irradiance, $\mu^{\alpha 0}$ is the transition electric dipole moment connecting the donor ground state and excited state, and $\rho_{f}^{A^{*}}$ is the density of states for the excited state, reflecting vibrational broadening. The dissipative effect of IVR does not feature in the electronic inter-state kinetics, though the associated red-shift in the emitted radiation is necessarily apparent and emerges in the following. Under normal conditions, i.e. below the saturation limit, $k_{A}^{k \leftarrow 0} \ll k_{A}^{0 \leftarrow 1}$ and equation (1) gives;

$$
\frac{N_{1 A}}{N_{A}} \simeq \frac{\pi I}{3 \hbar c \varepsilon_{0} k_{A}^{0 \leftarrow 1}}\left|\boldsymbol{\mu}^{\alpha 0(A)}\right|^{2} \rho_{f}^{A^{*}} .
$$

For the RET step, we have to consider both energy pooling transfer mechanisms. As shown in earlier work based on molecular $\mathrm{QED}^{15,16}$ the results emerge, from fourth-order time-dependent perturbation theory, in the form of the following rate equation;

$$
\begin{aligned}
\Gamma_{R E T}^{B \leftarrow A, A^{\prime}} & =\frac{2 \pi}{\hbar} \mid \mu_{i}^{0 \alpha(A)} V_{i j}(\mathbf{R}) \alpha_{j k}^{\beta 0(B)}(-\omega,-\omega) V_{k l}\left(\mathbf{R}^{\prime}\right) \mu_{l}^{0 \alpha\left(A^{\prime}\right)} \\
& +\mu_{i}^{0 \alpha(A)} V_{i j}\left(\mathbf{R}^{\prime \prime}\right) \alpha_{j k}^{0 \alpha\left(A^{\prime}\right)}\left(\omega^{\prime},-\omega\right) V_{k l}\left(\mathbf{R}^{\prime}\right) \mu_{l}^{\beta 0(B)} \\
& +\left.\mu_{i}^{0 \alpha\left(A^{\prime}\right)} V_{i j}\left(\mathbf{R}^{\prime \prime}\right) \alpha_{j k}^{0 \alpha(A)}\left(\omega^{\prime},-\omega\right) V_{k l}(\mathbf{R}) \mu_{l}^{\beta 0(B)}\right|^{2} \rho_{f}^{B} .
\end{aligned}
$$

Here the first term corresponds to cooperative transfer, while the further two terms relate to accretive transfer, and each is cast in Cartesian components using the implied summation convention for repeated indices $i, j, k$ and $l$. Also, $\omega$ and $\omega^{\prime}$ correspond to the donor emission circular frequencies, where $\omega \leq \omega_{0}$ and $\omega^{\prime} \leq 2 \omega_{0}$ due to excited state IVR, given that $\omega_{0}$ is the optical input frequency, and the donor-acceptor displacement vectors are defined as: $\mathbf{R}=\mathbf{R}_{B}-\mathbf{R}_{A}$, $\mathbf{R}^{\prime}=\mathbf{R}_{B}-\mathbf{R}_{A^{\prime}}$ and $\mathbf{R}^{\prime \prime}=\mathbf{R}_{A^{\prime}}-\mathbf{R}_{A}$. Finally, each $\alpha_{j k}$ is a generalized two-photon response tensor and $V_{i j}$ defines the electric dipole-electric dipole coupling tensor in the short-range region and is expressed, generally, by the following equation;

$$
V_{i j}(\mathbf{R})=\left(4 \pi \varepsilon_{0}\right)^{-1}\left(\delta_{i j}-3 \hat{R}_{i} \hat{R}_{j}\right) R^{-3}
$$

Note, equation (5) is a good approximation for the $V_{i j}$ tensor of the studied system, since within the chromophore array we have $R<<\hbar$ (where $\lambda=2 \pi / k$ designates the wavelength regime associated with energy transferal); for the representation of transfer between well-separated molecules, one would need to employ a more complex retarded E1-E1 coupling tensor ${ }^{21}-$ of which (5) is only the first term. Putting together the various factors delineated above, the ensemble averaged rate equation for the energy pooling process is as follows; 


$$
\begin{aligned}
\left\langle\Gamma^{\text {Pooling }}\right\rangle= & N_{A}\left(N_{A}-1\right)\left(\frac{\pi^{\frac{3}{2}}}{3 \hbar^{\frac{3}{2}} c \varepsilon_{0} k_{A}^{0 \leftarrow 1}}\right)^{2}\langle I\rangle^{2}\left|\boldsymbol{\mu}^{\alpha 0(A)}\right|^{4}\left(\rho_{f}^{A^{*}}\right)^{2} \rho_{f}^{B} \\
& \times \mid \mu_{i}^{0 \alpha(A)} V_{i j}(\mathbf{R}) \alpha_{j k}^{\beta 0(B)} V_{k l}\left(\mathbf{R}^{\prime}\right) \mu_{l}^{0 \alpha\left(A^{\prime}\right)}+\mu_{i}^{0 \alpha(A)} V_{i j}\left(\mathbf{R}^{\prime \prime}\right) \alpha_{j k}^{0 \alpha\left(A^{\prime}\right)} V_{k l}\left(\mathbf{R}^{\prime}\right) \mu_{l}^{\beta 0(B)} \\
& +\left.\mu_{i}^{0 \alpha\left(A^{\prime}\right)} V_{i j}\left(\mathbf{R}^{\prime \prime}\right) \alpha_{j k}^{0 \alpha(A)} V_{k l}(\mathbf{R}) \mu_{l}^{\beta 0(B)}\right|^{2} .
\end{aligned}
$$

Here for conciseness the frequency dependence of the $\alpha^{a b}$ factors is now implicit and follows from the superscripts. Equation (6) will serve as a basis for judging the relative efficiency of energy pooling and two-photon energy transfer mechanisms; the latter is the subject of the next section.

\section{TWO-PHOTON RESONANCE ENERGY TRANSFER PROCESSES}

TPRET is a process in which the two-photon excitation of a single donor is followed by conventional one-photon RET to the acceptor. To construct the rate equation for the complete process, a similar development to that used in the last section is employed. For the ensuing analysis the relevant population factor is simply the number of suitably excited donors within the focal volume, $N_{2 A}$. With steady-state conditions we have;

$$
\frac{N_{2 A}}{N_{A}} \simeq \frac{k_{A}^{2 \leftarrow 0}}{k_{A}^{0 \leftarrow 2}}
$$

where $k_{A}^{2 \leftarrow 0}$ and $k_{A}^{0 \leftarrow 2}$ are the two-photon absorption and de-excitation rate constants respectively - the former again derived from molecular QED in rotationally-averaged form as follows; ${ }^{22}$

$$
\begin{aligned}
\left\langle k_{A}^{2 \leftarrow 0}\right\rangle= & \frac{\left\langle\Gamma_{A}^{2 \leftarrow 0}\right\rangle}{N_{0 A}}=\frac{\pi\left\langle I^{2}\right\rangle}{120 \hbar}\left(\varepsilon_{0} c\right)^{-2}\left\{\left(2|\mathbf{e} \cdot \mathbf{e}|^{2}-1\right) \alpha_{i i}^{\alpha 0(A)}(\omega, \omega) \bar{\alpha}_{j j}^{\alpha 0(A)}(\omega, \omega)\right. \\
& \left.-\left(|\mathbf{e} \cdot \mathbf{e}|^{2}-3\right) \alpha_{i j}^{\alpha 0(A)}(\omega, \omega) \bar{\alpha}_{i j}^{\alpha 0(A)}(\omega, \omega)\right\} \rho_{f}^{A^{* * *}} .
\end{aligned}
$$

Here e.e is the self-product of the laser polarisation unit vector, which equates to 0 and 1 for circular and plane polarisations, respectively. For the single-step RET which delivers energy to the acceptor we have the familiar result from second-order time-dependent perturbation theory; ${ }^{23}$

$$
\Gamma_{R E T}^{B \leftarrow A}=\frac{2 \pi}{\hbar}\left|\mu_{i}^{0 \alpha(A)} V_{i j}(\mathbf{R}) \mu_{j}^{\beta 0(B)}\right|^{2} \rho_{f}^{B}
$$

Thus the total rate equation for a two-photon resonance energy transfer process emerges as;

$$
\begin{aligned}
\left\langle\Gamma^{T P R E T}\right\rangle & =\frac{N_{1 A} g_{11}^{(2)}}{60 k_{A}^{0 \leftarrow 2}}\left(\frac{\langle I\rangle \pi}{\varepsilon_{0} c \hbar}\right)^{2}\left\{\left(2|\mathbf{e} \cdot \mathbf{e}|^{2}-1\right) \alpha_{i i}^{\alpha 0(A)} \bar{\alpha}_{j j}^{\alpha 0(A)}-\left(|\mathbf{e} \cdot \mathbf{e}|^{2}-3\right) \alpha_{i j}^{\alpha 0(A)} \bar{\alpha}_{i j}^{\alpha 0(A)}\right\} \\
& \times\left|\mu_{i}^{0 \alpha(A)} V_{i j}(\mathbf{R}) \mu_{j}^{\beta 0(B)}\right|^{2} \rho_{f}^{A^{* * *}} \rho_{f}^{B},
\end{aligned}
$$

where $g_{11}^{(2)}$ is the single-site degree of second-order optical coherence, ${ }^{24}$ and again the frequency dependence of the molecular tensors is implicit. It is apparent that the only significant similarity between equations (6) and (10) is the 
shared feature of a quadratic dependence on mean irradiance. The relative efficiencies of the two mechanisms are determined by other factors, key amongst which are those relating to the detailed form and strengths of the electronic transitions.

\section{COMPARISON OF ELECTRONIC FACTORS}

Each of the mechanisms for optically nonlinear photoactivity entails a different form of interaction for the donor and acceptor units. The selection rules for one-photon and two-photon processes differ and, for a given system, one or more of the mechanisms or sub-mechanisms may be forbidden by local symmetry. The transition dipole moments $\mu^{a b}$ for single-photon processes are associated with the irreducible representation (irrep) $D^{(1-)}$ of an odd-parity rank 1 tensor, whilst for the two-photon interactions the representations of the even-parity rank 2 tensors $\alpha^{a b}$ comprise the irreps $D^{(0+)}$ $\oplus D^{(1+)} \oplus D^{(2+)} .25$ The (1+) irrep components of the two-photon tensors vanish only if the latter relate to two energetically equivalent photon events (e.g. the absorptions of two photons having identical frequency) - otherwise they remain, as for example in any resonance Raman scattering process. The detailed form of the irrep components is governed by the local point group symmetry, $D_{3 h}$ in many photoactive materials of dendrimeric form. ${ }^{26,27}$ The nature of the associated donor and acceptor transitions imposes conditions on the validity of each mechanism. Generally, the direct product of the initial and final state representations must span at least one of the irrep components of the relevant transition tensors. For example the accretive sub-mechanism is only symmetry-allowed if the donor decay transition has transformation properties replicated by a component of $D^{(1-)}$, i.e. the irrep for the initial photoabsorption. Furthermore, the same transition must have the transformation properties associated with a component of $D^{(0+)} \oplus D^{(1+)} \oplus D^{(2+)}$. The excitation transition dipole moment of the acceptor will also need to transform as one or more components of $D^{(1-)}$. Detailed analysis of the $D_{3 h}$ case reveals that when the direct product of the donor ground and excited state representations includes the irrep $E^{\prime}$, all of the discussed mechanisms are permitted; only cooperative pooling is allowed when the same product spans $A_{2}^{\prime \prime}$.

Despite the portrayal of many real dendrimeric and allied polymer materials as planar systems, attention must be paid to their actual flexibility and hence, their secondary structure and packing. ${ }^{28,29}$ In fact, many high-generation dendrimers are more closely biomimetic precisely because of their essentially globular habit - a facet hidden by the common graphical depictions. ${ }^{30}$ Globally, this will tend to obviate any symmetry in the complete dendrimer; locally, however, it may be expected that three-fold site symmetry will be retained for dendrimers based on 1,3,5-trisubstituted benzene. Unless there is any local twist in the structure, it is likely that the effect of folding will be to lower the site symmetry from $D_{3 h}$ to $C_{3 v}$. In this case, the irreps $E^{\prime}$ and $A_{2}^{\prime \prime}$ will correlate to $E$ and $A_{1}$, respectively, and corresponding conclusions can be drawn from the above $D_{3 h}$ analysis..

In general, the initiation of TPRET requires only one excited donor and the initial energy deposition is localised to that species. Often, photoactive materials are designed with spacer units separating the donors and acceptors, so as to retain their distinct electronic integrity and preclude charge transfer. However, if two or more identical donor species within the proximity of each other are electronically coupled to any significant extent, an exciton may form. Excitons in this context $^{31}$ are associated with an uncertainty in the location of the photon energy deposition and are generated when the number of excitations within a chromophore array is less than the number of donors it comprises. Hence energy pooling processes, which require two excited donor species, may engage three or more donors in excitonic states. Consider, for example, a threefold symmetric, nodal component of a dendrimer comprising chemically identical donors $\mathrm{A}, \mathrm{B}$ and $\mathrm{C}$ each at one corner of an equilateral triangle, with an acceptor, D, at the centre. Both in TPRET and energy pooling a donor exciton intermediate can form, as is illustrated by figure 2 and the equation;

$$
\mathrm{A}+\mathrm{B}+\mathrm{C}+\mathrm{D}+2 \hbar \omega \stackrel{\text { Exciton formation }}{\longrightarrow}(\mathrm{A}+\mathrm{B}+\mathrm{C})^{* * *}+\mathrm{D} \stackrel{R E T}{\longrightarrow} \mathrm{A}+\mathrm{B}+\mathrm{C}+\mathrm{D}^{*}
$$




\section{$3 S_{1}$}

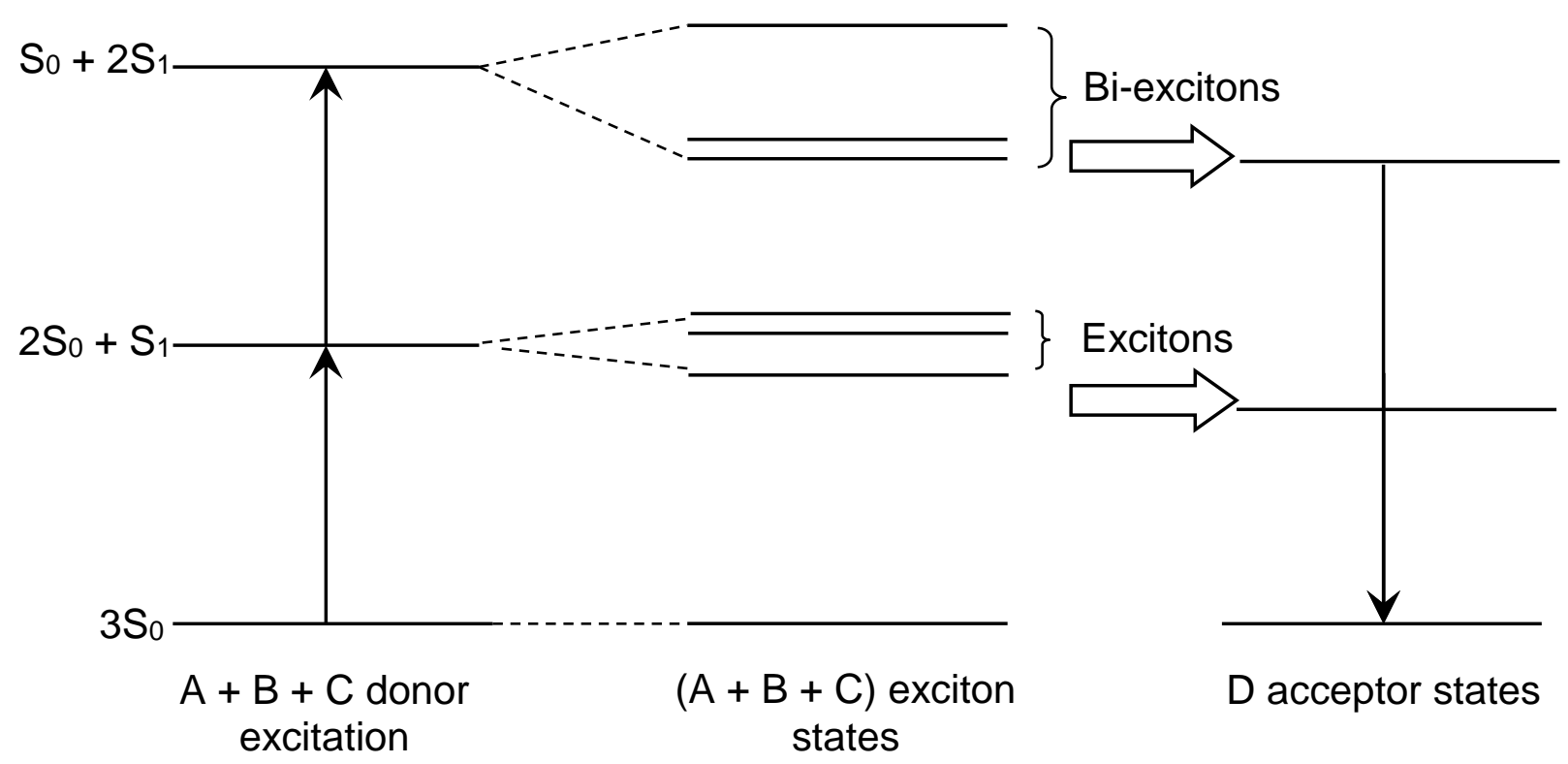

Fig. 2. Energy scheme for transfer of excitation energy from donor array exciton $(A+B+C)^{* *}$ to acceptor, $D$.

Although associated in each case with the energy of two input photons, the exciton is recognized to have a different structure for the two processes. Specifically, $(A+B+C)^{* *}$ is one of two forms - either $\left(A^{* *}+B+C\right)$ and its permutations for TPRET, or $\left(\mathrm{A}^{*}+\mathrm{B}^{*}+\mathrm{C}\right)$ and permutations for energy pooling. ${ }^{32,33}$ As the number of donors surrounding an acceptor on a given dendrimer node increases, the significance of excitonic transfer also grows, and increasingly favors the accretive mechanism - a point we shall return to in the discussion.

The detailed form of spectral overlap associated with each mechanism is a matter of considerable interest. As in conventional single-donor energy transfer, to determine a rate equation connected to Förster theory for TPRET mechanisms requires the consideration of spectral overlap between the donor fluorescence spectrum and the dispersive absorption cross-section of the acceptor - the detailed form depending on distance, and in the short range leading to the familiar Förster result. The QED formulation of this rate equation is given by; ${ }^{23}$

$$
\Gamma_{R E T}^{B \leftarrow A}=\frac{9}{8 \pi c^{2} \tau_{A}} \int_{0}^{\infty} F_{A}\left(\omega^{\prime}\right) \sigma_{B}\left(\omega^{\prime}\right) \omega^{\prime 2} g\left(\omega^{\prime}, \mathbf{R}\right) d \omega^{\prime}
$$

Equation (11) includes the radiative lifetime of the donor, $\tau_{A}$, the cross-section of the acceptor absorption and donor emission spectra, these are defined generally as; 


$$
\sigma_{B}(\omega)=\frac{\pi \omega}{3 \varepsilon_{0} c}\left|\boldsymbol{\mu}^{0 \beta(B)}\right|^{2} \sum_{m, p} \rho_{B}^{(m)}\left|\left\langle\varphi_{B^{*}}^{(p)} \mid \varphi_{B}^{(m)}\right\rangle\right|^{2} \delta\left(e_{B_{p}^{*}}-e_{B_{m}}-\hbar \omega\right),
$$

and

$$
F_{A}(\omega)=\frac{\omega^{3} \tau_{A}}{3 \varepsilon_{0} \pi \hbar c^{3}}\left|\mu^{\alpha 0(A)}\right|^{2} \sum_{n, r} \rho_{A^{*}}^{(n)}\left|\left\langle\varphi_{A}^{(r)} \mid \varphi_{A^{*}}^{(n)}\right\rangle\right|^{2} \delta\left(e_{A_{n}^{*}}-e_{A_{r}}-\hbar \omega\right),
$$

respectively. Here, $\rho$ is the population distribution function of the initial vibrational states of the specified species, each of the indices $n, r, m, p$ specifies the set of vibrational etc. sublevels of the transfer species, the energies of the initial and final state of each species are included in the energy-conserving delta function. In detail $F_{A}(\omega)$ is determined by the exit state of A, which is a consequence of initial excitation and subsequent IVR. Also included in equation (11) is $g\left(\omega^{\prime}, \mathbf{R}\right)$ which in the short-range (intramolecular) limit is $g\left(\omega^{\prime}, \mathbf{R}\right)=\left(c / \omega^{\prime} R\right)^{6} \eta_{3}^{2}$, where $\eta_{3}$ is an orientational factor;

$$
\eta_{3}=\left(\hat{\boldsymbol{\mu}}_{A} \cdot \hat{\boldsymbol{\mu}}_{B}\right)-3\left(\hat{\mathbf{R}} \cdot \hat{\boldsymbol{\mu}}_{A}\right)\left(\hat{\mathbf{R}} \cdot \hat{\boldsymbol{\mu}}_{B}\right)
$$

Hence we obtain;

$$
\Gamma_{R E T}^{B \leftarrow A}=\frac{9 c^{4} \eta_{3}^{2}}{8 \pi \tau_{A} R^{6}} \int_{0}^{\infty} F_{A}\left(\omega^{\prime}\right) \sigma_{B}\left(\omega^{\prime}\right) \omega^{\prime-4} d \omega^{\prime}
$$

The form of spectral overlap associated with the energy pooling processes, i.e. twin-donor transfer, is more complex due to the energy transferal of $\hbar \omega$ from $t w o$ donor species to one acceptor. The rate observable accommodates both transfer mechanisms and also their quantum interference as given by the following; ${ }^{34}$

$$
\begin{aligned}
\Gamma_{R E T}^{B \leftarrow A, A^{\prime}}= & \frac{2 \pi \rho_{f}^{B}}{\hbar}\left\{\left|M_{f i}^{a c c 1}\left(\mathbf{R}^{\prime}, \mathbf{R}^{\prime \prime}\right)\right|^{2}+\left|M_{f i}^{a c c 2}\left(\mathbf{R}, \mathbf{R}^{\prime \prime}\right)\right|^{2}+\left|M_{f i}^{c o o p}\left(\mathbf{R}, \mathbf{R}^{\prime}\right)\right|^{2}\right. \\
& +2 \operatorname{Re}\left(M_{f i}^{a c c 1}\left(\mathbf{R}^{\prime}, \mathbf{R}^{\prime \prime}\right) \bar{M}_{f i}^{a c c 2}\left(\mathbf{R}, \mathbf{R}^{\prime \prime}\right)+M_{f i}^{a c c 1}\left(\mathbf{R}^{\prime}, \mathbf{R}^{\prime \prime}\right) M_{f i}^{c o o p}\left(\mathbf{R}, \mathbf{R}^{\prime}\right)\right. \\
& \left.\left.+M_{f i}^{a c c 2}\left(\mathbf{R}, \mathbf{R}^{\prime \prime}\right) M_{f i}^{c o o p}\left(\mathbf{R}, \mathbf{R}^{\prime}\right)\right)\right\} .
\end{aligned}
$$

where $M_{f i}$ is the quantum amplitude of cooperative (coop) or accretive (accl, acc2) transfer-accl, acc 2 differentiating contributions which differ only through the interchange of A and $\mathrm{A}^{\prime}$. For example, the cooperative component of equation (16) (also signifying the expression for a system where selection rules preclude accretive transfer) is given by;

$$
\begin{aligned}
\left|M_{f i}^{\text {coop }}\left(\mathbf{R}, \mathbf{R}^{\prime}\right)\right|^{2} & =\frac{9}{64 \pi^{2} c^{4} \tau_{A} \tau_{A^{\prime}}} \iint \omega^{2}\left(\omega^{\prime}-\omega\right)^{2} F_{A}(\omega) F_{A^{\prime}}\left(\omega^{\prime}-\omega\right) \tilde{\sigma}_{B}\left(\omega, \omega^{\prime}-\omega\right) \\
& \times g(\omega, \mathbf{R}) g\left(\omega^{\prime}-\omega, \mathbf{R}^{\prime}\right) d \omega d \omega^{\prime}
\end{aligned}
$$

where $F_{A}$ and $F_{A^{\prime}}$ are both given by equation (13), and the cross-section of the acceptor two-photon absorption, $\tilde{\sigma}_{B}\left(\omega, \omega^{\prime}-\omega\right)$, is determined by writing the rate of two-photon absorption (TPA), as follows; 


$$
\begin{aligned}
\Gamma_{T P A} & =\frac{\pi \hbar n n^{\prime} \omega\left(\omega^{\prime}-\omega\right)}{2 \varepsilon_{0}^{2} V^{2}}\left|\boldsymbol{\alpha}^{B}\left(\omega, \omega^{\prime}-\omega\right)\right|^{2} \sum_{m, p} \rho_{B}^{(m)}\left|\left\langle\varphi_{B^{*}}^{(p)} \mid \varphi_{B}^{(m)}\right\rangle\right|^{2} \delta\left(e_{B_{p}^{*}}-e_{B_{m}}-\hbar \omega^{\prime}\right) \\
& =\frac{\tilde{\sigma}_{B}\left(\omega, \omega^{\prime}-\omega\right) n n^{\prime} c^{2}}{V^{2}} .
\end{aligned}
$$

Here $n$ and $n^{\prime}$ are photon numbers derived from number states and $V$ is the quantisation volume. Equation (18) can be rearranged to give;

$$
\tilde{\sigma}_{B}\left(\omega, \omega^{\prime}-\omega\right)=\frac{\pi \hbar \omega\left(\omega^{\prime}-\omega\right)}{2 \varepsilon_{0}^{2} c^{2}}\left|\alpha^{B}\left(\omega, \omega^{\prime}-\omega\right)\right|^{2} \sum_{m, p} \rho_{B}^{(m)}\left|\left\langle\varphi_{B^{*}}^{(p)} \mid \varphi_{B}^{(m)}\right\rangle\right|^{2} \delta\left(e_{B_{p}^{*}}-e_{B_{m}}-\hbar \omega^{\prime}\right)
$$

For the case where selection rules dictate that only accretive transfer occurs, three terms persist (accl, acc 2 and their interference) and the appropriate result can again be directly recovered from equation (16).

\section{DISCUSSION}

It is of interest to compare other features that distinguish the two main transfer mechanisms for energy pooling. These include; (i) quantum interference, whose manifestation is evident when considering the two transfer mechanisms for energy pooling, because the two electronic couplings that each of these involves are concerted and not step-wise processes, as the QED calculations show. In other words the quantum pathways from the initial to the final state traverse only virtual states, in which energy losses are not sustained into or beyond the femtosecond timescale; (ii) nanoscale architecture - it emerges that energy pooling processes in dendrimeric systems with a threefold symmetric nodal motif are generally dominated by the cooperative transfer mechanism due to the closer proximity of the donor and acceptor species in comparison to the donor-donor separation. This follows from the form of the coupling tensors in the two cases, $V_{i j}(\mathbf{R}) V_{k l}\left(\mathbf{R}^{\prime}\right)$ for the cooperative mechanism and $V_{i j}\left(\mathbf{R}^{\prime \prime}\right) V_{k l}\left(\mathbf{R}^{\prime}\right)$ for the accretive, bearing in mind that each $\mathbf{V}(\mathbf{R})$ has an overall dependence on $R^{-3}$, where $R$ is the magnitude of the appropriate displacement vector. In terms of this distance dependence, considered in isolation from other factors, the favorability of the accretive over the cooperative mechanism is enhanced as the number of donor molecules around the acceptor increases, by a factor that rises from 0.037 to 9.76 as the number of surrounding donors increases from three to nine. It is also of note that the two mechanisms are in this sense on an equal footing only when six donors surround an acceptor, as the donor-donor and donor-acceptor separations are then equal.

In this paper we have begun to address the principles associated with a multitude of factors whose interplay determines the favoured mechanism for optically nonlinear photoactivity. In general, nanomaterials of this kind are designed to expedite one specific mechanism. In most of the work that has concerned the characterisation of these systems, it has not been recognized that the two mechanisms can operate in parallel, given suitably placed chromophore energy levels, and subject to the geometric and symmetry-based criteria that we have delineated. Our results also emphasize the significance of quantum interferences within energy pooling framework. There is considerable scope to exploit this diversity of mechanisms, and it is our hope that as increasingly detailed principles emerge, these will inform and steer future efforts in the creation of photoactive nanomaterials.

\section{ACKNOWLEDGMENT}

DSB is funded by a studentship from the Engineering and Physical Sciences Research Council. 


\section{REFERENCES}

1. D. L. Andrews, "Optical energy harvesting materials", Introduction to Complex Mediums for Optics and Electromagnetics, ed. W. S. Weiglhofer and A. Lakhtakia, (SPIE, Bellingham, Washington, 2003) pp. 141-163.

2. A. Archut and F. Vögtle, "Functional cascade molecules", Chem. Soc. Rev. 27, 233-240 (1998).

3. A. Adronov and J. M. J. Fréchet, "Light-harvesting dendrimers", Chem. Commun., 1701-1710 (2000).

4. V. Balzani, P. Ceroni, M. Maestri and V. Vicinelli, "Light-harvesting dendrimers", Curr. Opin. Chem. Biol. 7, 657665 (2003).

5. N. Nishiyama, H. R. Stapert, G.-D. Zhang, D. Takasu, D.-L. Jiang, T. Nagano, T. Aida and K. Kataoka, "Light-harvesting ionic dendrimer porphyrins as new photosensitizers for photodynamic therapy", Bioconjug. Chem. 14, 58-66 (2003).

6. G.-D. Zhang, N. Nishiyama, A. Harada, D.-L. Jiang, T. Aida and K. Kataoka, "pH-sensitive assembly of light-harvesting dendrimer zinc porphyrin bearing peripheral groups of primary amine with poly(ethyleneglycol)-bpoly(aspartic acid) in aqueous solution”, Macromolecules 36, 1304-1309 (2003).

7. M. Halim, I. D. W. Samuel, J. N. G. Pillow and P. L. Burn, "Conjugated dendrimers for LEDs: control of colour", Synth. Met. 102, 1113-1114 (1999).

8. A. W. Freeman, S. C. Koene, P. R. L. Malenfant, M. E. Thompson and J. M. J. Fréchet, "Dendrimer-containing light-emitting diodes: toward site-isolation of chromophores", J. Am. Chem. Soc. 122, 12385-12386 (2000).

9. K. König, "Multiphoton microscopy in life sciences", J. Microscopy 200, 83-104 (2000).

10. D. J. S. Birch, "Multiphoton excited fluorescence spectroscopy of biomolecular systems", Spectrochimica Acta A 57, 2313-2336 (2001).

11. A. Adronov, J. M. J. Fréchet, G. S. He, K. S. Kim, S. J. Chung, J. Swiatkiewicz and P. N. Prasad, "Novel two-photon absorbing dendritic structures", Chem. Mater. 12, 2838-2841 (2000).

12. G. S. He, T. C. Lin, Y. Cui, P. N. Prasad, D. Brousmiche, J. M. Serin and J. M. J. Fréchet, "Two-photon excited intramolecular energy transfer and light-harvesting effect in novel dendritic systems", Opt. Lett. 28, 768-770 (2003).

13. D. Brousmiche, J. M. Serin, J. M. J. Fréchet, G. S. He, T. C. Lin, S. J. Chung and P. N. Prasad, "Fluorescence energy transfer in a novel two-photon absorbing system", J. Am. Chem. Soc. 125, 1448-1449 (2003).

14. O. Mongin, J. Brunel, L. Porrès and M. Blanchard-Desce, "Synthesis and two-photon absorption of triphenylbenzene-cored dendritic chromophores", Tetrahedron Lett. 44, 2813-2816 (2003).

15. R. D. Jenkins and D. L. Andrews, "Three-centre systems for energy pooling: quantum electrodynamical theory", $J$. Phys. Chem. A. 102, 10834-10842 (1998).

16. R. D. Jenkins and D. L. Andrews, "Twin-donor systems for resonance energy transfer", Chem. Phys. Lett. 301, 235-240 (1999).

17. R. D. Jenkins and D. L. Andrews, "Orientation factors in three-centre energy pooling", Phys. Chem. Chem. Phys. 2, 2837-2843 (2000).

18. G. J. Daniels and D. L. Andrews, "The electronic influence of a third body on resonance energy transfer", J. Chem. Phys. 116, 6701-6712 (2002).

19. P. Allcock and D. L. Andrews, "Two-photon fluorescence: resonance energy transfer", J. Chem. Phys. 108, 3089-3095 (1998).

20. P. Allcock and D. L. Andrews, "Two-photon fluorescence resonance energy transfer in protein-fluorophore complexes", Trends in Optics and Photonics Vol.22: Biomedical Optical Spectroscopy and Diagnostics/ Therapeutic Laser Applications, ed. E. M. Sevick-Muraca, J. A. Izatt and M. N. Ediger, (Optical Society of America, Washington, 1998) pp. 84-88.

21. G. J. Daniels, R. D. Jenkins, D. S. Bradshaw and D. L. Andrews, "Resonance energy transfer: The unified theory revisited", J. Chem. Phys. 119, 2264-2274 (2003). 
22. D. P. Craig and T. Thirunamachandran, Molecular Quantum Electrodynamics, (Dover, Mineola, NY, 1998) pp. 110-116.

23. D. L. Andrews and G. Juzeliūnas, "Intermolecular energy transfer: Retardation effects", J. Chem. Phys. 96, 66066612 (1992).

24. R. Loudon, The Quantum Theory of Light, 3rd edn, (University Press, Oxford, 2000) pp. 107-114.

25. D. L. Andrews, "Symmetry characterization in molecular multiphoton spectroscopy", Spectrochimica Acta 46A, 871-885 (1990).

26. M. R. Shortreed, S. F. Swallen, Z.-Y. Shi, W. Tan, Z. Xu, C. Devadoss, J. S. Moore and R. Kopelman, "Directed energy transfer funnels in dendrimeric antenna supermolecules ", J. Phys. Chem. B 101, 6318-6322 (1997).

27. Z. Xu and J. S. Moore, "Design and synthesis of a convergent and directional molecular antenna", Acta Polym. 45, 83-87 (1994).

28. J. R. Parquette, “The intramolecular self-organization of dendrimers”, C. R. Chimie 6, 779-789, 2003.

29. J. M. J. Fréchet, "Dendrimers and other dendritic macromolecules: From building blocks to functional assemblies in nanoscience and nanotechnology", J. Polymer Sci. A: Polymer Chem. 41, 3713-3725, 2003.

30. S. Thayumanavan, P. Bharathi, K. Sivanandan and D. R. Vutukuri, "Towards dendrimers as biomimetic macromolecules", C. R. Chimie 6, 767-778, 2003.

31. R. D. Jenkins and D. L. Andrews, "Multichromophore excitons and resonance energy transfer: molecular quantum electrodynamics", J. Chem. Phys. 118, 3470-3479, 2003.

32. R. D. Jenkins and D. L. Andrews, "Exciton resonance energy transfer: effects of geometry and transition moment orientation in model photosystems", Photochem. Photobiol. Sci. 2, 130-135, 2003.

33. R. D. Jenkins and D. L. Andrews, "Biexciton resonance energy transfer in a model photosystem", Photochem. Photobiol. Sci. 3, 39-46, 2004.

34. D. L. Andrews and R. D. Jenkins, "A quantum electrodynamical theory of three-centre energy transfer for upconversion and downconversion in rare earth doped materials", J. Chem. Phys. 114, 1089-1100, 2001.

*david.andrews@physics.org 